\title{
Análise ergonômica do livro Aprenda a Costurar com vistas para o aperfeiçoamento da aprendizagem da modelagem plana do vestuário
}

\author{
Ergonomic analysis of the book Learn to Sew with a focus on \\ improvement on learning process of flat cutting pattern making
}

ergonomia, usabilidade, modelagem do vestuário,ensino ergonomics, usability, pattern making, education

\begin{abstract}
Este artigo faz parte da dissertação de mestrado intitulada " 41 anos de estudos de modelagem do vestuário: uma proposta de aperfeiçoamento do ensino de modelagem através da usabilidade", cujo objetivo é verificar, através das heurísticas de Nielsen (1993), quais são as desconformidades ergonômicas do conteúdo apresentado no livro "Aprenda a Costurar", de Gil Brandão, escrito em 1967. A escolha pela obra que data de mais de quarenta anos justifica-se pelo fato de que até os dias atuais a mesma serve de suporte para o ensino e aprendizagem da modelagem plana do vestuário. A pesquisa se propõe a identificar oportunidades de melhorias no conteúdo do livro em questão com vistas para a usabilidade. No presente trabalho, utiliza-se o método de observação indireta, aplicando-se entrevista semi-estruturada com professores de modelagem como instrumento de coleta de dados. O resultado encontrado revela que o autor apresenta desconformidades ergonômicas nos seus textos, cada uma relativa a um critério de usabilidade.
\end{abstract}

This article is part of the master's research entitled " 41 years of garment modeling studies: one improvement purpose of modeling education through usability", whose objective is to verify, through the Nielsen heuristics (1993), what are the ergonomic discontinuities of the content presented in the book "Learn to Sew", by Gil Brandão, written in 1967. The choice of this work dating back more than forty years is justified by the fact that even nowadays it serves as a support for teaching and learning of the flat cutting pattern making. In this sense, the research aims to identify opportunities for improvement of the content in question with a focus on usability. In this paper, the method of indirect observation has been used, applying semi-structured interviews with pattern making teachers as a tool for data collection. The results obtained revealed that the author present ergonomic discontinuities in his text, each relating to a criterion of usability. 


\section{Introdução}

Este artigo apresenta dados sobre a análise ergonômica feita em um conteúdo específico contido no livro de Gil Brandão (Aprenda a Costurar), de 1967. Pretende-se identificar oportunidades de aperfeiçoamento do ensino e aprendizagem da modelagem com base nos critérios para a usabilidade. Apesar de que estes conceitos estejam associados primariamente a interfaces humano-computador (IHC), uma segunda vertente de estudo, apresentada neste trabalho, indica que a usabilidade é passível de ser associada a livros impressos como fator para a aprendizagem. Neste caso, adotou-se a obra citada em função de todo o reconhecimento que a mesma teve na década de sessenta, e, pelo seu teor de raridade, por não ser mais editado, o livro é um dos mais requisitados na área de modelagem plana e ainda é utilizado por professores e alunos nos dias atuais como livro didático referencial para o ensino da modelagem, bem como por modelistas autodidatas e profissionais da área.

Do ponto de vista acadêmico, para Hahn (1999), pedagogia e ergonomia, apesar de estarem inseridas em campos de estudo diferentes, possuem vários pontos em comum, especialmente em termos didáticos como conforto e facilidade na execução das tarefas e processamento de informações que visam relacionar o aluno ao conteúdo ministrado.

Durante a leitura e interpretação de conteúdos dos livros de modelagem, pode-se detectar falhas nos textos apresentados, ou seja, textos que não indicam claramente o passo a ser tomado pelo executor da tarefa e que apresentam o conteúdo sem estruturação e densidade adequada, deixando o leitor, ou aluno, confuso.

Geralmente, as falhas de interpretação da execução do diagrama da modelagem por estudantes de cursos de design de moda são decorrentes de uma disposição de palavras que não foi construída com o objetivo de facilitar a comunicação desta com o usuário. Exemplificando, pode-se comparar o texto da modelagem com uma interface gráfica computadorizada, no sentido explanado por Mager (2004): "o projeto de interface gráfica computadorizada deve se preocupar em estruturar o conteúdo, hierarquizando a informação de acordo com o objetivo da tarefa, desenvolvendo um bom layout para suas páginas com botões e ícones bem localizados e claros em seu significado”. Para Cybis (2007), existe uma 'configuração de base' que favorece a existência da usabilidade na relação usuário-sistema. Esta configuração é feita à medida que se respeitam os critérios, princípios ou heurísticas da usabilidade.

Os livros, neste sentido, podem ser comparados às interfaces de computador no que diz respeito aos critérios de usabilidade, ou seja, esta pesquisa parte do pressuposto de que também é possível fazer uma análise ergonômica de trechos de livros, ou obras completas, tal como é feita em softwares diversos. No caso dos livros, quando não há usabilidade, é possível que haja uma leitura mal feita do conteúdo, de modo a gerar dúvidas e bloqueios na comunicação entre aluno e objeto. 
Para ratificar a importância desta relação entre a usabilidade e livros de modelagem, bem como coletar informações importantes sobre a interação de alunos ou profissionais com os traçados da modelagem, a autora elaborou uma entrevista semi-estruturada, aplicada a professores de modelagem, e que trouxe os seguintes dados:

Quadro 1 Origem dos entrevistados (Fonte: autora)

\begin{tabular}{ll}
\hline Quantidade de Professores & Origem (Estado) \\
\hline 02 & RS \\
\hline 03 & $\mathrm{SC}$ \\
\hline 01 & $\mathrm{SP}$ \\
\hline 02 & $\mathrm{RJ}$
\end{tabular}

Sete dos oito entrevistados concordam que a ocorrência de erros cometidos pelos alunos pode ter relação com a maneira como a informação é transmitida pelos autores. As razões relatadas para estes erros estão apresentadas no seguinte gráfico, conforme respostas dos entrevistados:

Porcentagem de erros cometidos (Fonte: autora)

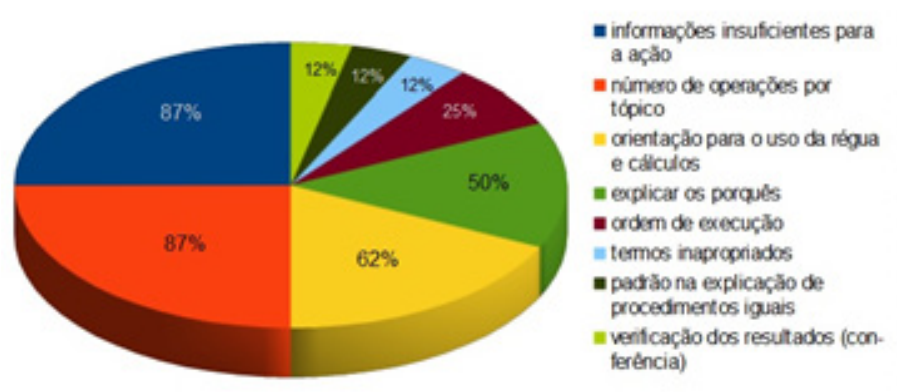

Cem por cento dos entrevistados concordam que uma análise ergonômica do traçado da modelagem é pertinente para melhorar a usabilidade dos livros de modelagem, e dentre as sugestões dadas a respeito de qual seria o conteúdo ideal do traçado da modelagem a fim de favorecer a aprendizagem e o controle de erros, constam:

1. utilizar uma linguagem mais descritiva para alunos iniciantes e uma mais sucinta para níveis avançados de conhecimento da modelagem;

2. organização das informações, conforme os princípios do design;

3. explicar a origem de cada medida e outros porquês da modelagem para que o leitor entenda o que está fazendo, em vez de reproduzir automaticamente um processo. Assim, o aluno torna-se autônomo na execução da tarefa. 
A eficiência do livro didático pode ser aumentada melhorando-se o nível de compreensão e retenção dos conhecimentos se estes forem apresentados em uma sequência lógica e com uma gradação progressiva das dificuldades (IIDA, 2005).

Sob a ótica da ciência, este trabalho é mais uma parte da construção do conhecimento recente e escasso, pois, não se tem relato de estudo de usabilidade acerca do conteúdo da modelagem, tampouco da obra de modelagem plana citada, especificamente. A secção do livro sobre a qual esta pesquisa se fundamenta, é a base do corpo da frente e seus respectivos diagramas, pois, uma vez sanados os problemas da base, tanto em termos de modelagem quanto de usabilidade, evita-se a sucessão de erros nos modelos subsequentes.

Por fim, este estudo está baseado nas dez heurísticas de usabilidade de Nielsen, contempladas em sua obra Usability Engineering (1993), por ser considerado um dos maiores especialistas em usabilidade nos Estados Unidos (CYBIS, 2007; PREECE, 2005) e por receber o maior número de citações na base de dados científicas brasileiras classificadas no Qualis da CAPES (Coordenação de Aperfeiçoamento de Pessoal de Nível Superior).

A presente pesquisa é de caráter qualitativo e de nível de interpretação descritivo, usado para descrever fenômenos e eventos existentes, identificar problemas e fazer comparações, visando esclarecer situações para futuras decisões (GRESSLER, 2007).

Os resultados desta pesquisa devem servir como um referencial para a elaboração futura de conteúdos de modelagem plana, sejam eles apostilas acadêmicas usadas por professores em instituições diversas, sejam livros didáticos produzidos por autores independentes.

\section{Ergonomia cognitiva}

Para Iida (2005), ergonomia é o estudo da interação entre o homem e o seu trabalho, equipamento e ambiente, através da aplicação dos conhecimentos de anatomia, fisiologia e psicologia na solução dos problemas surgidos dessa interação. Devido à sua amplitude, a ergonomia passou a ser tratada pela Associação Internacional de Ergonomia (IEA) em três campos distintos: ergonomia física, ergonomia organizacional e ergonomia cognitiva, salientando que essas são as macro-classificações, pois as mesmas podem ser subdivididas em domínios menores.

A ergonomia física está relacionada às características da anatomia humana, antropometria, fisiologia e biomecânica, na relação homematividade física.

A ergonomia organizacional lida com os sistemas sócio-técnicos, incluindo sua estrutura organizacional, política e de processos. Os tópicos relevantes incluem comunicação, organização do trabalho, cultura organizacional, gestão da qualidade, arranjo físico das instalações (layout), ética empresarial, liderança e trabalho em equipe. 
A ergonomia cognitiva, também chamada de engenharia psicológica, refere-se aos processos mentais, tais como a percepção de estímulos, o processamento e armazenamento de informações, memória, aprendizado, raciocínio, desempenho de habilidades e resposta motora, conforme interfiram nas relações entre homem e elementos de um sistema. O termo cognição é derivado da palavra latina cognitione, que significa a aquisição de um conhecimento através da percepção, ou o modo como o cérebro armazena toda a informação (STERNBERG, 2008).

De maneira simples, a ergonomia cognitiva ocorre da seguinte forma: o homem recebe um dado, que é processado em seu cérebro, transformando-se em uma informação. Esta informação gera uma ação, regida por dois fatores: a forma como o dado foi percebido e como o processo cognitivo se deu (ABRANTES, 2011). A ergonomia cognitiva também se apropria de diversos dados fundamentados na psicologia cognitiva e neurociências.

Logo, de acordo com Iida (2005) e Kroemer (2005), a ergonomia estuda não somente os aspectos físicos como os aspectos sensoriais dos sistemas em que o homem está inserido. Em se tratando da experiência da Interação Humano-Computador (IHC), os programas de software e suas interfaces constituem ferramentas cognitivas em virtude de serem capazes de produzir informações, criar representações, adicionar e subtrair dados (CYBIS, 2007). Sendo assim, para criar tais interfaces, estabelecem-se processos cognitivos humanos, que, segundo Preece (2005), são: atenção, percepção, memória, aprendizado, leitura (junto à fala e audição) e a resolução de problemas - planejamento, raciocínio e tomada de decisões.

\section{Usabilidade}

Usabilidade, de acordo com a Norma ISO 9241:10 e Preece (2004), é sinônimo de qualidade em uso e pode ser medida na extensão em que um produto seja fácil de usar, eficiente e agradável do ponto de vista do usuário, dentro de um determinado contexto. Ou seja, a usabilidade de um produto é afetada não somente pelas características do mesmo, mas pelo seu contexto de uso (MAGER, 2004). Ergonomia, segundo Cybis (2007, p. 173), é "a qualidade da adaptação de um dispositivo a seu operador e à tarefa que este realiza”.

Para Cybis (2007), a base da usabilidade é a integração entre interface, usuário, tarefa e ambiente, compondo o que Iida (2005) caracteriza como sistema: conjunto de elementos que interagem entre si com um objetivo comum e que evoluem no tempo.

Para Nielsen (1993), a usabilidade se aplica a todos os aspectos de um sistema com o qual o homem pode interagir, incluindo procedimentos de instalação e manutenção. A usabilidade é dividida em metas que tem por objetivo otimizar as interações estabelecidas 
entre produtos (ou programas) e pessoas, de modo a permitir que realizem atividades no trabalho, em casa e na escola. São elas:

1. learnability (ser fácil de aprender)

2. eficiência (ser eficiente no uso)

3. memorability (ser fácil de lembrar como se usa)

4. controle de erros (que sejam poucos e fáceis de solucionar)

5. satisfação (agradável ao usuário)

Shneiderman (1998) afirma que essas metas são conseguidas através de um planejamento cuidadoso, da sensibilidade às necessidades do usuário, da devoção à análise de requisitos e testes diligentes.

Segundo Cybis (2007), um problema de usabilidade ocorre durante a interação do sistema com o usuário, atrapalhando a realização da tarefa, mas ele se origina em um problema de ergonomia da interface. Portanto, pode-se dizer que por meio da avaliação da ergonomia, é possível chegar a um problema hipotético de usabilidade, que só será comprovado por meio de um teste de usabilidade. Logo, um problema de ergonomia é explícito quando identificado pela avaliação da ergonomia, ou implícito, quando sugerido por meio de um teste de usabilidade.

As técnicas de avaliação da ergonomia, de acordo com Cybis (2007, p.180), "são diagnósticas e se baseiam em verificações e inspeções de aspectos ergonômicos das interfaces que possam colocar-se como um problema ao usuário durante sua interação com o sistema". As desconformidades ergonômicas levam a três tipos de problemas de usabilidade, apontados por Cybis (2007):

1. barreira: aspecto da interface que impede a realização da tarefa e faz com que o usuário desista de utilizar uma função do sistema. As barreiras consomem tanto o tempo do usuário quanto do projetista, que desenvolveu uma função inutilizável;

2. obstáculo: aspecto da interface no qual o usuário se defronta com frequência, mas aprende a contorná-lo. De todo modo, um obstáculo sempre representa perda de desempenho na realização da tarefa;

3. ruído: aspecto que causa diminuição no desempenho da tarefa, apesar de não ser considerado barreira nem obstáculo. Em função de ruídos na interação, o usuário pode desenvolver aversão ao sistema, por exemplo, quando o sistema faz uma pergunta que exige que o usuário perca algum tempo pensando na resposta.

Assim, para que as metas de usabilidade sejam atingidas, evitando estes tipos de problema, os sistemas precisam ser desenvolvidos sob critérios ergonômicos, que foram estudados por diversos autores da área e que estão disponíveis em bibliografias variadas sobre 
usabilidade. Conforme descrito, neste trabalho foram adotadas as heurísticas de Nielsen (1993), apresentadas a seguir.

\section{As dez heurísticas de Nielsen}

As heurísticas de Nielsen serão tratadas à parte em função de a pesquisa ter sido conduzida baseada nas mesmas. O autor considera a usabilidade como sendo um dos aspectos que influenciam a aceitabilidade de um produto. Nielsen avalia a usabilidade sob dez heurísticas, retiradas de seu livro Usability Engineering (1993):

1. diálogo simples e natural: os usuários frequentemente escolhem funções do sistema por engano, e precisam de uma "saída de emergência”, visivelmente identificada, para deixar aquela situação indesejável sem ter que passar por um extenso diálogo;

2. falar a linguagem do usuário: o sistema deve falar a língua do usuário com palavras e conceitos familiares a este, em vez de adotar termos específicos da engenharia de software e outros sistemas;

3. minimizar a sobrecarga de memória: o sistema deve permitir que o usuário reconheça objetos, ações e opções em vez de precisar recuperá-los em sua memória;

4. consistência e padrões: a mesma informação deve ser sempre apresentada na mesma localização em todas as telas e caixas de diálogos e deve ser formatada do mesmo modo para facilitar o reconhecimento pelo usuário. Além disto, se o usuário sabe que o mesmo comando ou ação trará determinada resposta, ele se sentirá mais seguro ao usar o sistema;

5. feedback: o sistema deve sempre manter os usuários informados sobre o que está acontecendo, e fornecer um feedback adequado, dentro de um tempo razoável. o feedback não deve acontecer somente quando um erro ocorre, mas quando uma operação é realizada com sucesso;

6. evidenciar as saídas: a fim de que o usuário sinta-se no controle do diálogo, o sistema deve oferecer um meio fácil de sair de tantas situações quanto for possível. por exemplo, todas as caixas de diálogo devem conter um botão "cancelar" ou outra forma de saída fácil para levar o usuário ao estado anterior, bem como o botão "desfazer" para diversas operações. A visibilidade destes botões é importante para o caso de o usuário encontrar-se em um local desconhecido e sentir receio de perder dados se fizer algo incomum;

7. fornecer atalhos: os atalhos servem para tornar uma operação frequente mais rápida. Aceleradores típicos incluem abreviações de uma ou mais tarefas, ou sejam, uma tecla do teclado, um duplo-clique, bem como botões disponíveis em partes do diálogo onde eles provavelmente serão requisitados; 
8. mensagens de erro: diagnóstico e correção: as mensagens de erro devem ser numa linguagem clara, não codificada, indicar o problema e sugerir uma solução;

9. prevenir erros: melhor do que ter a mensagem de erro, é evitar o erro. Uma das maneiras é perguntar se o usuário realmente deseja executar tarefas como a exclusão de um arquivo. $\mathrm{O}$ sistema deve evitar conter comandos muito similares, ou comandos completamente diferentes reunidos. do mesmo modo, os diálogos não devem conter informações irrelevantes que induzam o usuário a percorrer o caminho errado;

10. fornecer ajuda e documentação das funcionalidades: mesmo que se busque desenvolver sistemas que sejam tão fáceis de manipular a ponto de não ser necessária qualquer ajuda ou documentação, nem sempre isto é possível. Porém, o manual nunca deve ser uma desculpa dada pelo designer quando o usuário considera uma interface difícil. Assim, qualquer informação deve ser fácil de buscar, focalizada na tarefa do usuário, além de listar passos concretos a serem executados e não ser muito longa.

\section{Análise ergonômica do livro de modelagem mediante as heurísticas de Nielsen}

Os princípios ergonômicos de Nielsen (1993) apresentados são destinados a interfaces humano-computador (IHC), portanto, alguns não podem ser adequadamente aplicados às características de um sistema tal como a execução da modelagem plana orientada por um livro, como é o caso dos critérios 6 e 7: evidenciar as saídas e fornecer atalhos, respectivamente. Isto posto, ambos foram descartados da análise do livro. Obteve-se, assim, oito princípios passíveis de serem relacionados com o conteúdo da modelagem presente nos livro:

Quadro 2 Heurísticas de Nielsen aplicáveis ao livro de modelagem (Fonte: autora)

\begin{tabular}{|l|}
\hline 1.Diálogo Simples e Natural \\
\hline 2.Falar a Linguagem do Usuário \\
\hline 3.Minimizar a Sobrecarga de Memória \\
\hline 4.Consistência e Padrões \\
\hline 5.Feedback \\
\hline 6.Mensagens de Erro: diagnóstico e correção \\
\hline 7.Prevenir Erros \\
\hline 8.Fornecer Ajuda sobre as Funcionalidades \\
\hline
\end{tabular}


Será feita, a seguir, a análise do traçado da base da frente da blusa de Gil Brandão, retirado de seu livro Aprenda a Costurar (1967), mediante os oito critérios definidos acima. Serão apontados tanto os aspectos positivos quanto os negativos do conteúdo. Abaixo, é apresentada uma imagem do conteúdo interno do livro que foi analisado.

Figura 1 Conteúdo do traçado da frente da blusa (Fonte: Brandão, 1967).
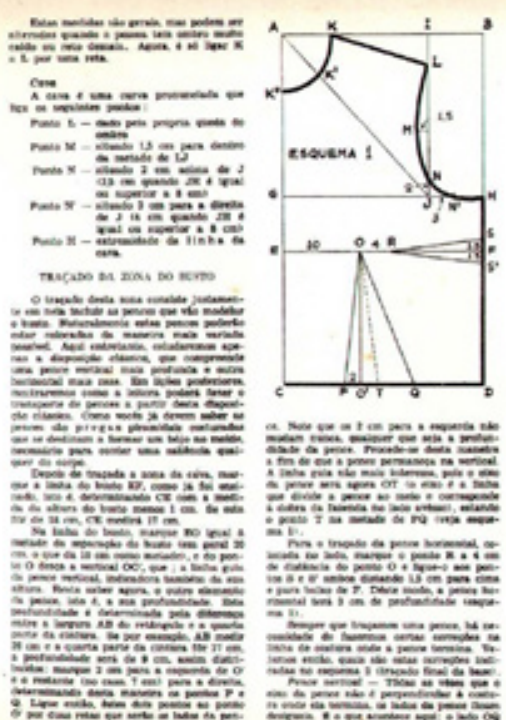
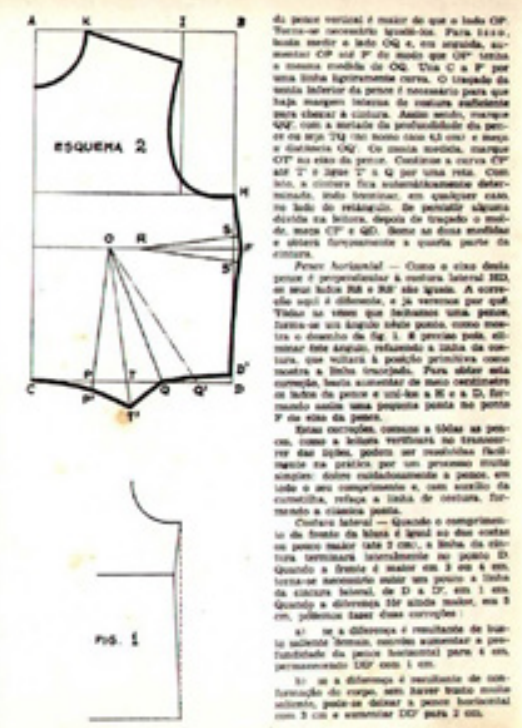

\subsection{Critério 1: Diálogo Simples e Natural}

O autor, por diversas vezes no decorrer do seu traçado, aponta a parte do corpo que será iniciada e o momento em que é finalizada, procurando manter uma conversa informal com o leitor. Neste caso, ele adota o termo "leitora", pois trata-se de uma obra escrita na época em que o ofício de corte-e-costura era destinado a mulheres.

No primeiro momento, na primeira página do traçado, o autor divide a base em três zonas (zona do busto, zona neutra e zona da cava), depois retoma a zona do busto e a zona da cava, não citando mais a zona neutra. Na segunda página do traçado, ao explicar a zona do busto, o autor repete uma informação já dada, a de como encontrar a linha do busto. Um problema sequencial ocorre quando o autor orienta a marcar um ponto sobre uma diagonal que ainda não foi traçada (AJ), sendo que o ponto J também não foi citado anteriormente. Deste modo, o leitor se vê obrigado a procura a diagonal e o ponto J no diagrama desenhado. Em termos gráficos, o autor utiliza as letras do alfabeto ordenadamente para marcar e nominar cada ponto no desenho. A fim de facilitar o entendimento, quando um seguimento exige mais do que dois pontos, como a curva do 
decote, o autor utiliza a mesma letra, acrescida de apóstrofos. Ex: K, K' e K”. Porém, não é o que ocorre na curva da cava, onde são usadas letras sequenciais diferentes e somente a letra $\mathrm{N}$ se repete com apóstrofo.

A respeito dos princípios gráficos estruturais do texto e das leis da Gestalt, observa-se que:

a) o autor não diferencia, algumas vezes, título de subtítulo: ambos em caixa alta, com a mesma fonte e tamanho;

b) não agrupa elementos que fazem parte da mesma informação. Pelas leis de proximidade, segregação e semelhança, da Gestalt, Gomes Filho (2000) aponta que elementos próximos uns dos outros tendem a ser vistos juntos, compondo unidades dentro do todo, bem como a igualdade de formas e cores, assim formando agrupamentos. No exemplo abaixo, por mais que o autor tenha escrito o decote e o ombro em parágrafos separados, estes elementos são de difícil percepção na visualização geral do texto.

c) raramente utiliza tópicos e ícones. A densidade do texto do autor faz com que as palavras estejam de tal forma agrupadas a ponto de comporem um único bloco, sendo difícil segregar algum elemento que o leitor esteja buscando, como um ponto, ou seguimento. Nas partes do texto em que predominam os tópicos, a localização de alguma orientação torna-se facilitada:

\subsection{Critério 2: Falar a Linguagem do Usuário}

Por se tratar de um livro escrito na década de 60, alguns substantivos não são mais apropriados para o linguajar da atualidade, como é o caso da palavra fazenda, citada diversas vezes pelo autor a fim de designar o que se conhece, hoje, por tecido.

\subsection{Critério 3: Minimizar a Sobrecarga de Memória}

Este é um aspecto bastante recorrente no diálogo do autor: há excesso de informações contidas no mesmo parágrafo, bem como muitas orientações dadas ao mesmo tempo para a execução de apenas uma tarefa.

\subsection{Critério 4: Consistência e Padrões}

De maneira geral, o autor procura manter o padrão na orientação de tarefas semelhantes. No entanto, em seu texto descritivo, o autor explica separadamente como corrigir cada uma das pences, vertical e horizontal, e, no parágrafo seguinte, o autor explica que 
todas as demais pences das lições subsequentes podem ser corrigidas de outra maneira através de um processo prático, também descrito no texto, mas que é diferente das duas primeiras.

\subsection{Critério 5: Feedback}

Este é um dos critérios mais presentes nas obras de Gil Brandão, pois, a cada nova ação, o autor informa quais resultados o leitor obterá. Em se tratando da meta de aprendizagem da usabilidade (learnability), o modo como ele transmite as informações aproxima o leitor do objeto de estudo (a modelagem) e permite que o mesmo entenda o conteúdo em vez de somente reproduzir um ato mecanizado. Com relação ao feedback em função de uma ação irreversível, o autor orienta o leitor a extrair o molde da base com o papel dobrado ao meio, a fim de obter o molde inteiro, sem que haja a necessidade de emendar no centro da frente.

\subsection{Critério 6: Mensagens de Erro: diagnóstico e correção}

Conforme já mencionado, o livro não tem a capacidade de responder a uma ação executada pelo leitor, mas pode ajudá-lo a conferir se seu traçado foi executado corretamente, de modo a propor, por exemplo, que se $x=1$, então $y=2$. Gil Brandão não destina uma parte específica do seu texto à conferência final do traçado para diagnosticar e corrigir erros, mas durante o texto, ele recomenda que o leitor confira certas medidas, a fim de garantir que uma ação complexa tenha sido executada corretamente.

\subsection{Critério 7: Prevenir Erros}

Em diversos momentos do texto de Gil Brandão, o autor exemplifica cálculos utilizando medidas comuns, a fim de que o leitor entenda a orientação com mais facilidade e consiga executar o mesmo cálculo com medidas diferentes de maneira mais eficaz. Pelo linguajar descritivo e detalhado, o autor também é enfático em determinadas orientações para que, mesmo o leitor tendo usado medidas diferentes, o traçado não fique comprometido. O autor não exemplifica a posição correta para o uso de réguas, mas menciona expressões como "curva suave" e "curva pronunciada".

\subsection{Critério 8: Fornecer Ajuda sobre as Funcionalidades}

No decorrer de seu texto, o autor costuma citar capítulos ou "lições" anteriores, ou que ainda estão por vir, para indicar que detalhes de determinado procedimento estão disponíveis no livro, 
caso o leitor deseje ler ou recordar. Gil Brandão também dispõe nas páginas iniciais de seu livro diversas tabelas de referência que são abordadas no decorrer do traçado da base da frente. Sem o conhecimento destas tabelas, o leitor passa a não executar algumas ações por falta de dados. Da mesma forma, está disponível a tabela de medidas, os desenhos das respectivas partes do corpo e os procedimentos de como tirar as medidas do corpo.

Após ter sido feita a avaliação ergonômica do traçado, apresenta-se um quadro-resumo que visa identificar, numa visão geral, os pontos fortes e fracos do conteúdo mediante as heurísticas, e o resultado é uma listagem de características desejáveis para a elaboração de um novo sistema, neste caso um possível novo traçado, bem como de aspectos desfavoráveis, os quais deverão ser evitados.

Quadro 3 Pontos fortes e fracos do conteúdo do livro mediante as heurísticas. Fonte: autora

\begin{tabular}{|c|c|}
\hline HEURÍSTICAS & RESULTADO DA ANÁLISE \\
\hline 1. Diálogo Simples e Natural & $\begin{array}{l}\text { - É repetitivo } \\
\text { - Comete erros de sequência } \\
\text { - Texto denso } \\
\text { - Apresentação gráfica ruim }\end{array}$ \\
\hline 2. Falar a Linguagem do Usuário & - Termos coloquiais \\
\hline 3. Minimizar a Sobrecarga de Memória & - Muitas ações em um só tópico \\
\hline 4. Consistência e Padrões & $\begin{array}{l}\text { - Mistura textos densos com tópicos e quadros } \\
\text {-Varia a forma de explicar }\end{array}$ \\
\hline 5. Feedback & $\begin{array}{l}\text { - Contextualiza o leitor } \\
\text { - Informa constantemente sobre o início e fim } \\
\text { de novas tarefas, justifica as ações. }\end{array}$ \\
\hline $\begin{array}{l}\text { 6. Mensagens de Erro: diagnóstico e } \\
\text { correção }\end{array}$ & $\begin{array}{l}\text { - Convida o leitor a conferir as medidas e } \\
\text { explica como corrigir erros detectados }\end{array}$ \\
\hline 7. Prevenir Erros & $\begin{array}{l}\text { - Não orienta no uso das réguas } \\
\text { - Exemplifica os cálculos }\end{array}$ \\
\hline 8. Fornecer Ajuda Sobre as Funcionalidades & $\begin{array}{l}\text { - Não omite informações } \\
\text { - Dedica páginas iniciais às tabelas, materiais e } \\
\text { tiragem de medidas } \\
\text { - Não contém dicionário de termos } \\
\text {-Não aborda conceitos de geometria básica } \\
\text { para as tarefas }\end{array}$ \\
\hline
\end{tabular}

\section{6 . Conclusão}

A conclusão deste trabalho parte da verificação de algumas questões levantadas, como os objetivos e o problema de pesquisa. Além de todo o referencial teórico, o processo de coleta de dados 
lervolino, F. | Análise ergonômica do livro Aprenda a Costurar

Infodesıın | São Paulo | v. 12 | n. 1 [2015], p. 128 - 142 
pelo qual esta pesquisa passou, trouxe importante contribuição para a abordagem do assunto, pouco explorado cientificamente, no sentido de mostrar que há, de fato, problemas ergonômicos nos materiais didáticos do ensino da modelagem, e que os mesmos podem ser resolvidos. Além disso, os dados coletados mostraram que há poucas discrepâncias de opinião quanto a estas desconformidades, dando sustentação e teor de pertinência à pesquisa dentro do ensino da modelagem.

A metodologia adotada contribuiu para tornar possível a análise ergonômica de interfaces não-computacionais, ou seja, a interface do livro de modelagem escolhido para este estudo: Aprenda a Costurar (BRANDÃO, 1967).

O que se percebe é que as dez heurísticas de Nielsen (1993), ainda que tenham sido sintetizadas para oito, em função de uma adaptação necessária às interfaces não-computacionais tratadas, demonstraram ser um conjunto de critérios eficaz na resolução do problema de pesquisa, que consistia em responder quais eram as desconformidades quanto à ergonomia do traçado da base da frente do autor.

Deste modo, conclui-se que Brandão (1967) atende aos requisitos de feedback; mensagens de erro: diagnóstico e correção, e falar a linguagem do usuário. O autor deixa a desejar no atendimento aos requisitos de prevenir erros e fornecer ajuda sobre as funcionalidades.

\section{Referências}

ARAÚJO, MÁRIO. 1996. Tecnologia do vestuário. Lisboa: Fundação Calouste Gulbenkian.

BRANDÃO, GIL. 1967. Aprenda a costurar. Rio de Janeiro : Edições Jornal do Brasil. CYBIs, W. 2007. Ergonomia e usabilidade: conhecimentos, métodos e aplicações.

São Paulo : Novatec Editora.

FIALHO, FRANCISCO A. P. 2001. Ciências da cognição. Florianópolis : Insular. GARDNer, H. 1994. Estruturas da Mente: A Teoria das Inteligências Múltiplas. Porto Alegre : Ed. Artes Médicas Sul.

GRESSLER, LORI ALICE. 2007. Introdução à Pesquisa: projetos e relatórios. São Paulo : Loyola.

GRIZ, CRISTINA, CARVALHO, GISELE DE, PEIXOTO, ANGÉLIKA. 2007. Ergonomia Cognitiva: a combinação de diversos recursos didáticos no ensino do Desenho Arquitetônico. In : Proceedings of the 11th Iberoamerican Congress of Digital Graphics. Anais... México.

Grossman, T; Fitzmaurice, G; ATtar, R. 2009. A Survey of Software Learnability: metrics, methodologies and guidelines. Boston (USA).

HAHN, TÂNIA MARIA. 1999. Por uma pedagogia ergonômica: mais cidadania no mundo do trabalho. Dissertação (Mestrado em Engenharia de Produção). Programa de Pós-Graduação em Engenharia de Produção. Florianópolis: UFSC, :134.

IIDA, ITIRO. 2005. Ergonomia: projeto e produção. São Paulo : Ed. Edgard Blucher $2^{\mathrm{a}}$ ed. 
JONES, SUE JENKYN. 2005. Fashion design: manual do estilista. Tradução de Iara Biderman. São Paulo: Cosac Naify.

KROEMER, K.H.E., GRANDJEAN, E. 2005. Manual de Ergonomia: adaptando o trabalho ao homem. Porto Alegre : Ed. Bookman - 5 $5^{\text {a }}$ ed.

MAGER, G. 2004. Interface gráfica para aplicativo computacional: desenvolvimento de uma interface baseada em critérios de Ergonomia, Usabilidade e Design. 2004. Dissertação (Mestrado em Engenharia de Produção) UFSC-SC, Florianópolis.

MARCONI, MARIA DE ANDRADE; laKatos, eVA MARIA. 1999. Técnicas de Pesquisa. São Paulo: Atlas.

MAYER, E. RICHARD. 1977. Cognição e Aprendizagem Humana. São Paulo :

Ed. Cultrix.

NIELSEN, J. 1993. Usability Engineering. São Francisco (EUA) : Morgan Kaufmann. NIELSEN, J. 2002. Homepage: 50 Websites desconstruídos. Rio de Janeiro : Campus. OliVeira, MARTA KOHL. 1998. Vygotsky: Aprendizado e desenvolvimento: Um processo sócio-histórico. São Paulo: Scipione.

PREECE, J. 2005. Design de interação: além da interação homem-computador. Porto Alegre : Bookman.

SHNEIDERMAN, B. 1998. Designing the User Interface: Strategies for Effective Human-Computer Interaction. 3. ed. EUA: Addison Wesley.

Sternberg, robert j. Psicologia Cognitiva. Porto Alegre $:$ Ed. Artmed $-4^{\underline{a}}$ ed, 2008.

WAll, P.; MARCuSso, N.; Telles, M. 2006. Tecnologia e Aprendizagem: tópicos de integração. Bases para a integração da tecnologia com a pedagogia. Vol 1. Coleção Tecnologia e Educação. São Paulo : Praxis.

\section{Sobre a autora}

\section{Fernanda Iervolino,}

Mestra em Design, UFSC. É professora do curso de Design da Universidade Federal de Santa Catarina (UFSC), departamento de Expressão Gráfica, atuando na área de desenvolvimento de produto com ênfase em desenho técnico e artístico de moda, modelagem plana e moulage e sistemas CAD/CAM para a indústria do vestuário. <fernanda.i@ufsc.br>

Artigo recebido em 22 mar. 2015, aprovado em 22 abr. 2015. 